\title{
TEM Investigations of Ion-Irradiated Cerium Oxide Thin Film
}

\author{
Sergei Rouvimov ${ }^{1}$ and Khachatur V. Manukyan ${ }^{2}$
}

1. Department of Electrical Engineering, University of Notre Dame, Notre Dame, IN, USA.

2. Department of Physics, University of Notre Dame, Notre Dame, IN, USA.

Cerium oxide $\left(\mathrm{CeO}_{2}\right)$ is of great interest due to its unique properties. The formation of a single oxygenvacancy during the reduction of $\mathrm{CeO}_{2}$ to $\mathrm{Ce}_{2} \mathrm{O}_{3}$ increases the $\mathrm{Ce}^{3+}$ fraction $[1,2,3]$. The valence and defect structures of $\mathrm{CeO}_{2}$ are dynamic and can change spontaneously or in response to physical parameters such as temperature, partial oxygen pressure, doping with other ions, and on applying an electric field or surface stress. Nano-scale $\mathrm{CeO}_{2}$ particles possess an increased $\mathrm{Ce}^{3+}$ concentration in comparison to larger particles since the defect concentration is augmented at the surface. The simultaneous presence of $\mathrm{Ce}^{3+}$ and $\mathrm{Ce}^{4+}$ mixed valence states impart properties of ceria that have been deployed in various fields such as fuel cell materials, automotive catalysis, oxygen storage, and biological reactive oxygen species scavenging [4,5].

It this work, energetic argon ion beams was used to tailor the structure of polycrystalline $\mathrm{CeO}_{2}$ thin films with a thickness of 15-20 nm deposited on the silicon wafer. A 5MV single ended (Sta Ana) NEC Pelletron and accelerator was used for producing argon ion beams with a different energy, flux, and fluence. By changing the irradiation parameters, it is possible to regulate the number of oxygen vacancies and the $\mathrm{Ce}^{3+} / \mathrm{Ce}^{4+}$ ratio in ceria thin films. The focus-ion-beam-milling technique was used to prepare electron transparent samples for imaging by transmission electron microscope (TEM). FEI Titan 80-300 TEM has been employed for atomic resolution imaging of the irradiated films. Electron diffraction and electron energy loss spectroscopy (EELS) were used to investigate the composition of films and valance state of cerium and oxygen.

Detailed TEM analysis suggested that ion irradiation significantly change the morphology and the structure of $\mathrm{CeO}_{2}$ layer. For example, initial non-irradiated film images display some pores, while irradiated samples result in reduction of porosity (Figure 1). Other significant observation is the thickness of amorphous interlayer between $\mathrm{CeO}_{2}$ and $\mathrm{Si}$ increases. Local high resolution EDS analysis show that that amorphous layer contains silicon, oxygen and cerium. High-resolution TEM imaging showed that a portions of $\mathrm{CeO}_{2}$ layer also amorphized suggesting that ion irradiation initiates intermixing of $\mathrm{CeO}_{2}$ and $\mathrm{Si}$ at the interface. ELLS measurements indicates that concentration of $\mathrm{Ce}^{3+}$ significantly increases upon ion irradiation suggesting intermixing of and amorphization leads to partial reduction of cerium at the interface.

References:

[1] TXT Sayle et al., Chem. Mater. 28 (2016), p. 728.

[2] T Naganuma and E Traversa, Nanoscale 16 (2012), p. 4950.

[3] D Widmann and RJ Behm, Acc. Chem. Res. 47 (2014), p. 740.

[4] D Yoon et al., Phys. Chem. Chem. Phys. 15 (2013), p. 14966.

[5] A Varma et al., Chem. Rev. 116 (2016), p. 14493. 


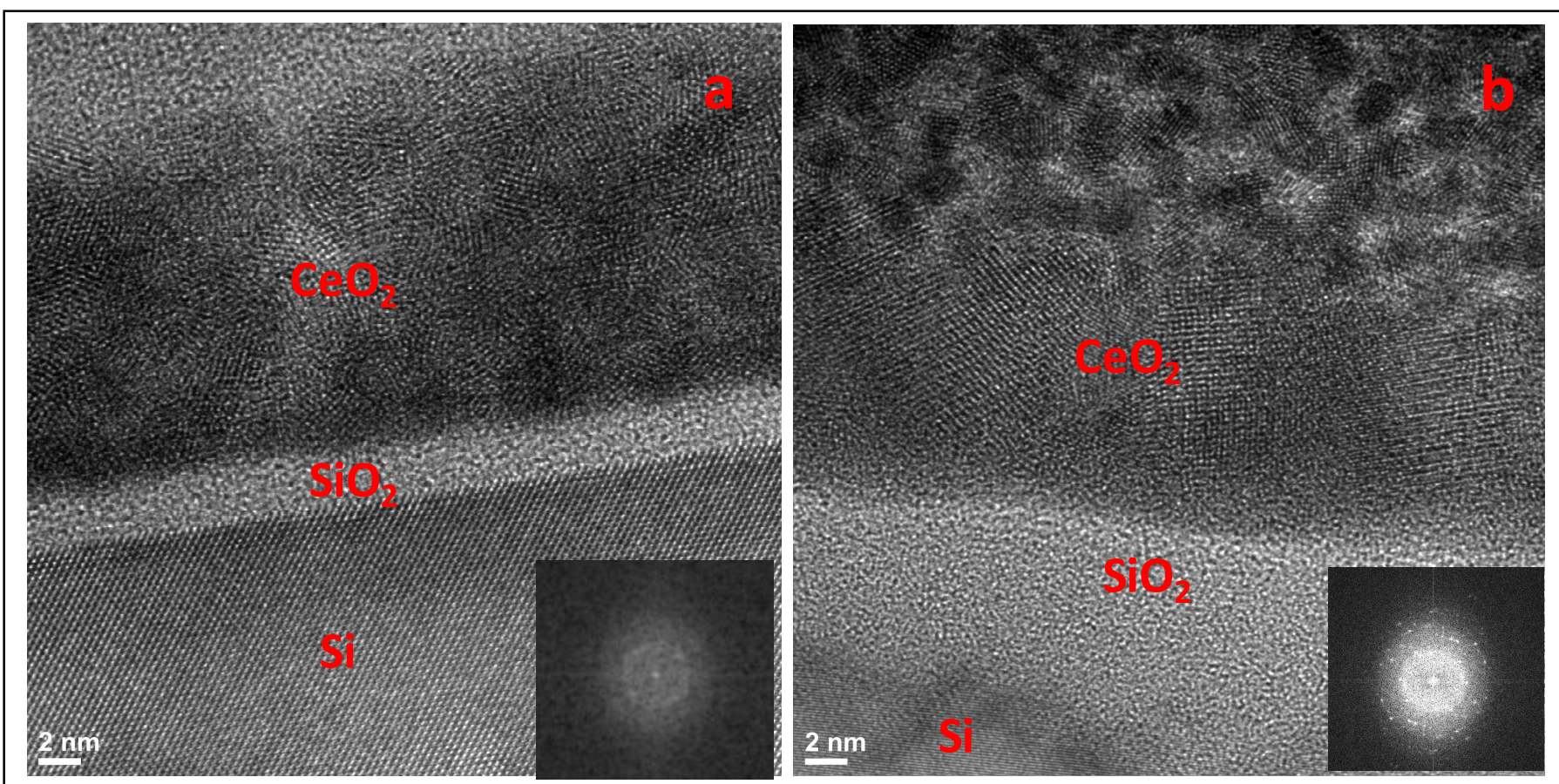

Figure 1. High-resolution TEM images of initial (a) and irradiated $\mathrm{CeO}_{2} / \mathrm{Si}$ (b) samples. $\left(1 \cdot 10^{16}\right.$ ion $/ \mathrm{cm}^{2}$ irradiation dose). Inserted are FFT of $\mathrm{CeO}_{2}$ crystalline areas.
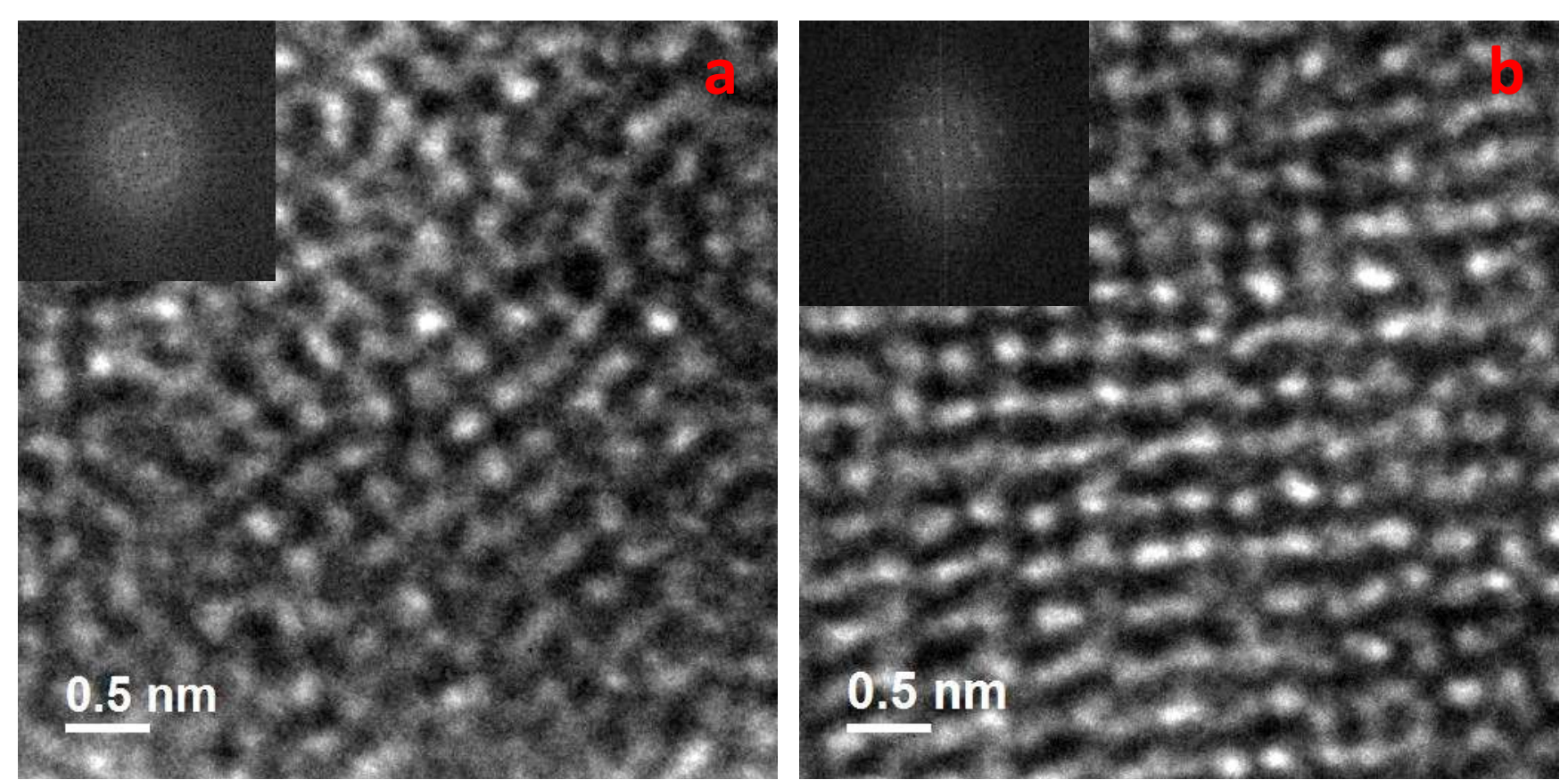

Figure 2. Enlarged sections of $\mathrm{CeO}_{2}$ crystalline areas in high-resolution TEM images of initial (a) and irradiated (b) $\mathrm{CeO}_{2} / \mathrm{Si}$ samples. $\left(1 \cdot 10^{16} \mathrm{ion} / \mathrm{cm}^{2}\right.$ irradiation dose) shown in Figure 1. 